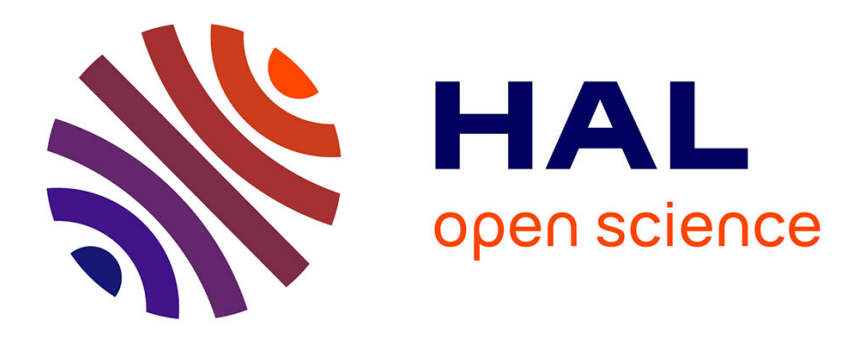

\title{
Safe 3D Bipedal Walking through Linear MPC with 3D Capturability
}

Adrien Pajon, Pierre-Brice Wieber

\section{To cite this version:}

Adrien Pajon, Pierre-Brice Wieber. Safe 3D Bipedal Walking through Linear MPC with 3D Capturability. ICRA 2019 - International Conference on Robotics and Automation, May 2019, Montréal, Canada. pp.1404-1409, 10.1109/ICRA.2019.8794117 . hal-02177530

\section{HAL Id: hal-02177530 \\ https://hal.science/hal-02177530}

Submitted on 9 Jul 2019

HAL is a multi-disciplinary open access archive for the deposit and dissemination of scientific research documents, whether they are published or not. The documents may come from teaching and research institutions in France or abroad, or from public or private research centers.
L'archive ouverte pluridisciplinaire $\mathbf{H A L}$, est destinée au dépôt et à la diffusion de documents scientifiques de niveau recherche, publiés ou non, émanant des établissements d'enseignement et de recherche français ou étrangers, des laboratoires publics ou privés. 


\title{
Safe 3D Bipedal Walking through Linear MPC with 3D Capturability
}

\author{
Adrien Pajon ${ }^{1}$, Pierre-Brice Wieber ${ }^{1}$
}

\begin{abstract}
We propose a linear MPC scheme for online computation of reactive walking motions, necessary for fast interactions such as physical collaboration with humans or collision avoidance in crowds. Unlike other existing schemes, it provides fully adaptable height, adaptable step placement and complete kinematic and dynamic feasibility guarantees, making it possible to walk perfectly safely on a piecewise horizontal ground such as stairs. A linear formulation is proposed, based on efficiently bounding the nonlinear term introduced by vertical motion, considering two linear constraints instead of one nonlinear constraint. Balance and Passive Safety guarantees are secured by enforcing a 3D capturability constraint. Based on a comparison between CoM and CoP trajectories involving exponentials instead of polynomials, this capturability constraint involves a CoM motion stopping along a segment of line, always maintaining complete kinematic and dynamic feasibility.
\end{abstract}

\section{INTRODUCTION}

Fast interactions such as physical collaboration with humans [1] or collision avoidance in crowds [2] require biped robots that are able to compute reactive walking motions completely online, quickly and reliably. In such cases, the safety of the computed motion in terms of balance and collision mitigation is naturally a crucial property to guarantee. A typical approach for walking robots is to make sure that they always engage in capturable trajectories, ending with a dynamically balanced and collision-free stopping motion [3]. This provides guarantees both for balance [4] and Passive Safety, making sure that the robot is always able to stop safely before any collision happens [5], [6].

The concept of boundedness has been proposed to provide a more general balance guarantee than capturability [7], not imposing that the robot has to be able to stop. But being able to stop is a key requirement of Passive Safety, and as a result, capturability appears to be more suitable to guarantee the safe navigation of legged robots in dynamic environments. This guides our choice to consider a capturability and not a boundedness property, although we don't address directly here the issue of collision mitigation.

Walking on a non-horizontal ground, e.g. slopes and stairs, can be approached with a linear dynamical model by maintaining a constant height of the Center of Mass $(\mathrm{CoM})$ of the robot above the ground [8] or a constant height of the corresponding Virtual Repellent Point (VRP) above the ground [9]. But demanding scenarios can require varying these heights, generally introducing nonlinearities. Developing fast and reliable numerical methods for generating walking motions with varying height led to various

This work has been funded by the EU H2020 Comanoid Research and Innovation Action (RIA).

${ }^{1}$ Adrien Pajon and Pierre-Brice Wieber are with Univ. Grenoble Alpes, Inria, 38000 Grenoble, France. adrien.pajon@gmail.com conservative approximations of these nonlinearities [7], [10], [11]

We adopt here the linear Model Predictive Control (MPC) scheme developed in [12], for its many desirable features. It provides automatic step placement, which is necessary to generate fully reactive walking motions as in [1] and [2]. It is formulated as a linear problem which can be solved very efficiently with off-the-shelf solvers, by bounding nonlinearities conservatively (making sure to maintain kinematic and dynamic feasibility) in a very effective way: the CoM and Center of Pressure $(\mathrm{CoP})$ trajectories obtained with this approach have been observed to be only a few millimeters away from the true optimum [13]. Comparable schemes typically consider predefined vertical motion [14] or predefined step placement [15] and don't provide complete kinematic and dynamic feasibility guarantees [7], which are all significant limitations.

Our main goal in this paper is to provide balance and Passive Safety guarantees by introducing a 3D capturability constraint, missing in the original MPC scheme proposed in [12]. We establish this capturability constraint based on a comparison between $\mathrm{CoM}$ and $\mathrm{CoP}$ trajectories involving exponentials as in [9] instead of polynomials as in [12]. This capturability constraint involves stopping along a segment of line as in [16], but considering only planar contact with the ground during the stopping motion to obtain a formulation which depends linearly on foot placement and CoM motion. Stopping along curves has already been approached, but with ad-hoc numerical schemes that can't be integrated in a linear MPC scheme as done here [17], [18].

This paper is structured as follows: Section II reviews $\mathrm{CoM}$ and CoP trajectories for walking on piecewise horizontal ground. Section III details dynamic and kinematic feasibility constraints related to walking. Section IV introduces 3D capturability. Section V provides implementation details regarding the linear MPC scheme. Section VI presents numerical results. Section VII concludes the paper.

\section{COM AND COP TRAJECTORIES FOR WALKING ON A PIECEWISE HORIZONTAL GROUND}

We consider a robot walking on a piecewise horizontal ground such as stairs. Considering zero angular momentum, the motion of the CoM $c$ of the robot can be related to the $\mathrm{CoP} p$ of the contact forces with the ground in the following way [12]:

$$
p^{x, y}=c^{x, y}-\zeta \ddot{c}^{x, y}
$$

with

$$
\zeta=\frac{c^{z}-p^{z}}{\ddot{c}^{z}+g}
$$


where $g$ is the vertical acceleration due to gravity, ${ }^{x}$ and $y$ refer to horizontal coordinates while ${ }^{z}$ refers to the vertical coordinate. When computing trajectories of the CoM of the robot on intervals of time $\left[t_{k}, t_{k+1}\right]$, solutions are usually based on exponentials as in [8], [9] or polynomials as in [1], [12].

\section{A. Piecewise exponential solutions}

Piecewise exponential solutions are typically of a form:

$$
c(t)=\alpha_{k}+\beta_{k} \tau_{k}+\gamma_{k} e^{-\lambda \tau_{k}}+\delta_{k} e^{\lambda \tau_{k}}
$$

with $\tau_{k}=t-t_{k}$ and constant parameters $\alpha_{k}, \beta_{k}, \gamma_{k}, \delta_{k}$ and $\lambda$. In this case, the CoM acceleration has a form:

$$
\ddot{c}(t)=\lambda^{2}\left(c(t)-\alpha_{k}-\beta_{k} \tau_{k}\right),
$$

which can be interpreted as a spring with stiffness $\lambda^{2}$ pushing the CoM away from a VRP moving linearly with time, as developped in [9]. We use the speed of the VRP $\beta_{k}$ to parameterize the trajectory of the CoM and take advantage of the remaining parameters $\alpha_{k}, \gamma_{k}$ and $\delta_{k}$ to enforce continuity in position, velocity and acceleration.

The interest of this type of trajectories is that if the VRP is kept at a constant height $\frac{g}{\lambda^{2}}$ above the ground with

$$
\alpha_{k}^{z}=p_{k}^{z}+\frac{g}{\lambda^{2}} \text { and } \beta_{k}^{z}=0
$$

then

$$
\zeta=\frac{1}{\lambda^{2}}
$$

and the CoP follows a straight line on the ground

$$
p_{k}^{x, y}(t)=\alpha_{k}^{x, y}+\beta_{k}^{x, y} \tau_{k}
$$

We consider varying the height of the VRP. In that case, it is proposed in [14], [15] to vary $\lambda$ accordingly so that (6) and (7) always hold, but this results in a nonlinear problem. We propose instead to keep $\lambda$ constant. In that case, (6) and (7) don't always hold.

\section{B. Piecewise polynomial solutions}

Piecewise polynomial solutions are typically of a form:

$$
c(t)=\alpha_{k}^{\prime}+\beta_{k}^{\prime} \tau_{k}+\frac{\gamma_{k}^{\prime}}{2} \tau_{k}^{2}+\frac{\delta_{k}^{\prime}}{6} \tau_{k}^{3}
$$

with constant parameters $\alpha_{k}^{\prime}, \beta_{k}^{\prime}, \gamma_{k}^{\prime}$ and $\delta_{k}^{\prime}$. In this case, the CoM acceleration has a form:

$$
\ddot{c}(t)=\gamma_{k}^{\prime}+\delta_{k}^{\prime} \tau_{k},
$$

which can be interpreted as the action of a simple force varying linearly with time. We use the jerk of the CoM $\delta_{k}^{\prime}$ to parameterize its trajectory and take advantage of the remaining parameters $\alpha_{k}^{\prime}, \beta_{k}^{\prime}$ and $\gamma_{k}^{\prime}$ to enforce continuity in position, velocity and acceleration.

\section{DYNAMIC AND KINEMATIC CONSTRAINTS}

\section{A. Dynamic feasibility}

Due to the unilaterality of contact forces, their CoP must lie in the contact surface [19]:

$$
p^{x, y} \in s_{i}^{x, y}+\mathcal{S}
$$

with $s_{i}$ the position of the ankle on the ground and $\mathcal{S}$ the shape of the sole. Following [12], we impose that the vertical motion of the CoM satisfies

$$
\underline{\zeta}\left(\ddot{c}^{z}+g\right) \leq c^{z}-p^{z} \leq \bar{\zeta}\left(\ddot{c}^{z}+g\right)
$$

with fixed bounds $\underline{\zeta}$ and $\bar{\zeta}$, so that we have

$$
\underline{\zeta} \leq \zeta \leq \bar{\zeta}
$$

assuming no free falling:

$$
\ddot{c}^{z}+g \geq 0 \text {. }
$$

This way, we know from (1) that

$$
p^{x, y} \in\left[c^{x, y}-\bar{\zeta} \ddot{c}^{x, y}, c^{x, y}-\underline{\zeta}^{x, y}\right],
$$

so a sufficient condition to satisfy 10 is

$$
\left\{c^{x, y}-\bar{\zeta} \ddot{c}^{x, y}, c^{x, y}-\underline{\zeta}^{x, y}\right\} \in s_{i}^{x, y}+\mathcal{S} .
$$

This approach allows considering two linear constraints 11 and $[15)$ instead of the nonlinear constraint $[10]$.

\section{B. Kinematic feasibility}

Following [12], we assume that the CoM of the robot is approximately at a constant position with respect to the hips. Considering the maximum leg length between the hips and ankles, we can conclude that the reachable region for the CoM is approximately a sphere defined with respect to the position of the ankle on the ground $s_{i}$. We consider a conservative polyhedral approximation of this sphere:

$$
A_{i}\left(c-s_{i}\right) \leq b_{i}
$$

with a matrix $A_{i}$ and vector $b_{i}$ depending on the support foot $i$. This polyhedral constraint must naturally be satisfied with respect to both feet on the ground during double support. We also constrain the respective positions of consecutive steps in order to avoid overlap:

$$
\underline{S}^{x, y} \leq s_{i+1}^{x, y}-s_{i}^{x, y} \leq \bar{S}^{x, y}
$$

\section{3D CAPTURABILITY}

Our main goal in this paper is to provide balance and Passive Safety guarantees by introducing a 3D capturability constraint, imposing that the robot always engages in trajectories ending with a dynamically balanced and collision-free stopping motion. During the interval of time $t \in\left[t_{M},+\infty[\right.$, where $t_{M}$ marks the end of the prediction horizon, we consider a trajectory based on exponentials as before:

$$
c(t)=\alpha_{M}+\beta_{M} \tau_{M}+\gamma_{M} e^{-\lambda \tau_{M}}+\delta_{M} e^{\lambda \tau_{M}} .
$$

But we need $\beta_{M}=\delta_{M}=0$ to obtain a trajectory

$$
c(t)=\alpha_{M}+\gamma_{M} e^{-\lambda \tau_{M}}
$$

that eventually comes to a stop as desired, at a position $\alpha_{M}$. To ensure continuity of the CoM trajectory at the end of the 
prediction horizon, we need to have

$$
\left[\begin{array}{l}
c\left(t_{M}\right) \\
\dot{c}\left(t_{M}\right) \\
\ddot{c}\left(t_{M}\right)
\end{array}\right]=\left[\begin{array}{c}
\alpha_{M}+\gamma_{M} \\
-\lambda \gamma_{M} \\
\lambda^{2} \gamma_{M}
\end{array}\right]
$$

Hence, we have

$$
\alpha_{M}=c\left(t_{M}\right)+\frac{\dot{c}\left(t_{M}\right)}{\lambda} \text { and } \gamma_{M}=-\frac{\dot{c}\left(t_{M}\right)}{\lambda}
$$

and we need to satisfy

$$
\ddot{c}\left(t_{M}\right)=-\lambda \dot{c}\left(t_{M}\right) .
$$

We obtain a linear motion of the CoM with velocity and acceleration in opposite directions, as in [16]. Thanks to the single exponentional in (19), both $c(t)$ and $\ddot{c}(t)$ are monotonous, so the dynamic and kinematic feasibility constraints need to be verified only at both ends of the interval. When $t=t_{M}$, this is done as previously in Section III, and when $t \rightarrow+\infty$, we have

$$
\left[\begin{array}{c}
c(t \rightarrow+\infty) \\
\dot{c}(t \rightarrow+\infty) \\
\ddot{c}(t \rightarrow+\infty)
\end{array}\right]=\left[\begin{array}{c}
\alpha_{M} \\
0 \\
0
\end{array}\right]
$$

so constraint (11) becomes

$$
g \underline{\zeta} \leq \alpha_{M}-p^{z} \leq g \bar{\zeta},
$$

constraint (15) becomes

$$
\alpha_{M}^{x, y} \in \operatorname{conv}\left\{s_{M}\right\},
$$

and constraint $(16)$ becomes

$$
A_{M}\left(\alpha_{M}-s_{M}\right) \leq b_{M} .
$$

\section{LINEAR MPC SCHEME}

Following [12], we define now a linear MPC scheme to generate 3D walking motions.

\section{A. Objectives}

The main objective of the walking motion is to follow a horizontal reference speed of the CoM $\dot{c}_{r e f}^{x, y}$, minimizing the deviation

$$
d_{1}=\mu_{1}\left\|\dot{c}^{x, y}-\dot{c}_{r e f}^{x, y}\right\|^{2}
$$

with a weight $\mu_{1}$. Energy consumption and walking speed on uneven ground can be improved by stretching knees and keeping the CoM high above the ground. A simple approach is to minimize the deviation from a reference height $h_{r e f}$ with a weight $\mu_{2}$ :

$$
d_{2}=\mu_{2}\left\|c^{z}-\left(s_{i}^{z}+h_{r e f}\right)\right\|^{2} .
$$

Robustness to disturbances is usually improved if the CoP is kept close to the center of the support foot. Considering the inclusion (14), we minimize the deviation between the center of the segment $\left[c^{x, y}-\bar{\zeta} \ddot{c}^{x, y}, c^{x, y}-\underline{\zeta}^{x, y}\right]$ and the center of the foot, which is at a position $\sigma_{i}$ with respect to the support ankle $s_{i}$ :

$$
d_{3}=\mu_{3}\left\|c^{x, y}-\frac{1}{2}(\bar{\zeta}+\underline{\zeta}) \ddot{c}^{x, y}-\left(s_{i}^{x, y}+\sigma_{i}^{x, y}\right)\right\|^{2}
$$

with a weight $\mu_{3}$.

As discussed in Section II] exponential solutions are parameterized by the speed of the VRP $\beta_{k}$ while polynomial solutions are parameterized by the jerk of the CoM $\delta_{k}^{\prime}$. A simple way to obtain smooth trajectories, which are generally desirable for complex mechanical systems such as humanoid robots, is to penalize these parameters. This corresponds to minimizing

$$
d_{4}= \begin{cases}\mu_{4}\left\|\beta_{k}\right\|^{2} & \text { for exponential solutions, } \\ \mu_{4}\left\|\delta_{k}^{\prime}\right\|^{2} & \text { for polynomial solutions }\end{cases}
$$

with a weight $\mu_{4}$.

\section{B. Quadratic Program}

The decision variables for our MPC scheme are the foot placements on the ground $s_{i}^{x, y}$ and depending on the choice of exponential or polynomial solutions, the speed of the VRP $\beta_{k}$ or the jerk of the CoM $\delta_{k}^{\prime}$ on each interval of time $\left[t_{k}, t_{k+1}\right]$. The dynamic and kinematic constraints (15)(17) and the capturability constraints (22)-(26) are all linear, while objectives (27)-(30) are all quadratic functions of these decision variables. Our MPC scheme can be formulated therefore as a Quadratic Program (QP):

$$
\begin{aligned}
\operatorname{minimize} & \sum_{k=m}^{M} d_{1}\left(t_{k}\right)+d_{2}\left(t_{k}\right)+d_{3}\left(t_{k}\right)+d_{4}\left(t_{k}\right) \\
\text { subject to } & 15-17 \text { at all } t_{k} \in\left[t_{m}, t_{M}\right], \\
& 22-126 \text { at } t_{M},
\end{aligned}
$$

where $m$ and $M$ indicate the beginning and end of the prediction horizon.

\section{NUMERICAL RESULTS}

We use a model of the HRP-4 [20] with weights

$$
\mu_{1}=1, \mu_{2}=10^{-2}, \mu_{3}=10^{-1} \text { and } \mu_{4}=10^{-7} \text {. }
$$

The prediction horizon covers 2 steps, each composed of $0.7 \mathrm{~s}$ of single support (SS) and $0.1 \mathrm{~s}$ of double support (DS). During each step, the DS is covered by 1 interval of $0.1 \mathrm{~s}$ while the $\mathrm{SS}$ is covered by $N$ intervals of $\frac{0.7}{N} \mathrm{~s}$. Hence, $M-m=2(N+1)$. We consider the following bounds on the height of the CoM above the ground:

$$
0.53 \leq c^{z}-p^{z} \leq 0.83,
$$

and a hexagonal pyramid for approximating the reachable region in 16. The shape of the soles $\mathcal{S}$ is rectangular, considering a $2 \mathrm{~cm}$ margin to account for the effect of a nonzero angular momentum on the CoP. Finally, we consider the following bounds on the nonlinear term:

$$
0.0521 \leq \zeta \leq 0.1173
$$

\section{A. Choice of $\lambda$}

The choice of the constant $\lambda$ is naturally guided by equations (6) and (7). We have observed that this choice is not so strict in our approach, although extreme values can 


\begin{tabular}{|c|c|c|}
\hline & exponential & polynomial \\
\hline$N=7$ & 3.3 & 2.6 \\
$N=5$ & 3.5 & 3.1 \\
$N=4$ & 3.1 & 3.1 \\
$N=2$ & 4.9 & 6.4 \\
$N=1$ & 3.6 & 9.8 \\
\hline
\end{tabular}

TABLE I: Maximum deviation in $\mathrm{mm}$ from a straight line of CoP trajectories based on exponentials or polynomials when walking on a flat ground, depending on the number $N$ of time intervals during single support.

lead to an infeasible set of constraints. We decide to use

$$
\lambda=\sqrt{\frac{g}{0,78}} \approx 3.54 .
$$

\section{B. Exponential or polynomial solutions}

The usual interest of exponential trajectories of the form (3) is that when the VRP is kept at a constant height $\frac{g}{\lambda^{2}}$ above the ground, the CoP follows a straight line on the ground. This simplifies significantly the constraint (10), that the $\mathrm{CoP}$ remains in the contact surface. On the contrary, in the case of polynomial trajectories of the form (8), the CoP follows a curve which deviates slightly from a straight line, what must be accounted for.

When the height of the CoM and VRP above the ground varies, the trajectory of the CoP deviates from a straight line in both cases. The size of this deviation naturally depends on the length of the intervals of time $\left[t_{k}, t_{k+1}\right]$. Considering that the single support is covered by $N$ intervals of $\frac{0.7}{N} \mathrm{~s}$, we can see in Table I that there is no clear difference between trajectories based on exponentials and polynomials, unless $N=2$ or 1 , with intervals of 0.35 or $0.7 \mathrm{~s}$. This result doesn't vary qualitatively with walking speed or step height.

We can see in Fig. 1 that when walking at 0.2 and $0.6 \mathrm{~m} . \mathrm{s}^{-1}$ on a flat ground, vertical motion is degraded when $N=2$ or 1 , and walking upstairs can even be infeasible. A choice of $N>4$ seems preferable if we want the vertical motion not constrained unnecessarily, and in that case, there is no visible difference between trajectories based on exponentials and polynomials.

\section{Capturability}

The addition of a capturability constraint in the end of the prediction horizon aims at guaranteeing balance and passive safety by always computing trajectories that end with a dynamically balanced stopping motion. In order to demonstrate this point, let's compare in Fig. 2 a view from above of the motion generated by the MPC scheme introduced in the previous Section, with and without this capturability constraint, over a typical prediction horizon starting in the middle of a single support phase while walking at $0.2 \mathrm{~m} . \mathrm{s}^{-1}$ on a horizontal ground.

We can see in Fig. 2b that when the capturability constraints (21)-26 are all enforced, the final exponential motion (18) is dynamically balanced and the CoM stops above the final support foot as desired. In this case, capturability of the generated motion is guaranteed. On the contrary, in Fig. 2a where only constraint 20 is enforced to maintain continuity of position and speed of the CoM, the final exponential motion (18) is clearly not dynamically balanced and the CoM doesn't even stop above the final support foot. In this situation, capturability of the generated motion is not guaranteed.

The capturability constraints 21- 26 have been designed to enable varying height of the CoM. This is demonstrated in Fig. 3 when walking up stairs of varying height $(19.5,18$, $18,18,14.5 \mathrm{~cm})$ at $0.6 \mathrm{~m} . \mathrm{s}^{-1}$.

\section{CONCLUSION}

We propose a linear MPC scheme for online computation of reactive walking motions on a piecewise horizontal ground such as stairs, with guarantees for both balance and Passive Safety. Unlike other existing schemes, it provides fully adaptable height, adaptable step placement and complete kinematic and dynamic feasibility guarantees, all in a single linear formulation, based on efficiently bounding the nonlinear term $\zeta$ introduced by vertical motion, considering two linear constraints $[11$ and $[15$ instead of a nonlinear constraint 10].

The balance and Passive Safety guarantees are secured by enforcing a 3D capturability constraint. Based on a comparison between CoM and CoP trajectories involving exponentials instead of polynomials, this capturability constraint involves a CoM motion stopping along a segment of line, always maintaining complete kinematic and dynamic feasibility. Numerical results are provided, and experiments with a real HRP-4 robot should hopefully follow soon.

\section{REFERENCES}

[1] D. J. Agravante, A. Sherikov, P.-B. Wieber, A. Cherubini, and A. Kheddar, "Walking pattern generators designed for physical collaboration," in Robotics and Automation (ICRA), 2016 IEEE International Conference on. IEEE, 2016, pp. 1573-1578.

[2] N. Bohórquez, A. Sherikov, D. Dimitrov, and P.-B. Wieber, "Safe navigation strategies for a biped robot walking in a crowd," in Humanoid Robots (Humanoids), 2016 IEEE-RAS 16th International Conference on. IEEE, 2016, pp. 379-386.

[3] T. Koolen, T. De Boer, J. Rebula, A. Goswami, and J. Pratt, "Capturability-based analysis and control of legged locomotion, part 1 : Theory and application to three simple gait models," The International Journal of Robotics Research, vol. 31, no. 9, pp. 1094-1113, 2012.

[4] M. Ciocca, P.-B. Wieber, and T. Fraichard, "Strong recursive feasibility in model predictive control of biped walking," in IEEE-RAS International Conference on Humanoid Robots, 2017.

[5] K. Macek, D. A. V. Govea, T. Fraichard, and R. Siegwart, "Towards safe vehicle navigation in dynamic urban scenarios," Automatika, 2009

[6] S. B. Liu, H. Roehm, C. Heinzemann, I. Lütkebohle, J. Oehlerking, and M. Althoff, "Provably safe motion of mobile robots in human environments," in Intelligent Robots and Systems (IROS), 2017 IEEE/RSJ International Conference on. IEEE, 2017, pp. 1352-1357.

[7] A. Zamparelli, N. Scianca, L. Lanari, and G. Oriolo, "Humanoid gait generation on uneven ground using intrinsically stable mpc," IFACPapersOnLine, vol. 51, no. 22, pp. 393-398, 2018.

[8] S. Kajita and K. Tani, "Study of dynamic biped locomotion on rugged terrain-derivation and application of the linear inverted pendulum mode," in Robotics and Automation, 1991. Proceedings., 1991 IEEE International Conference on. IEEE, 1991, pp. 1405-1411. 


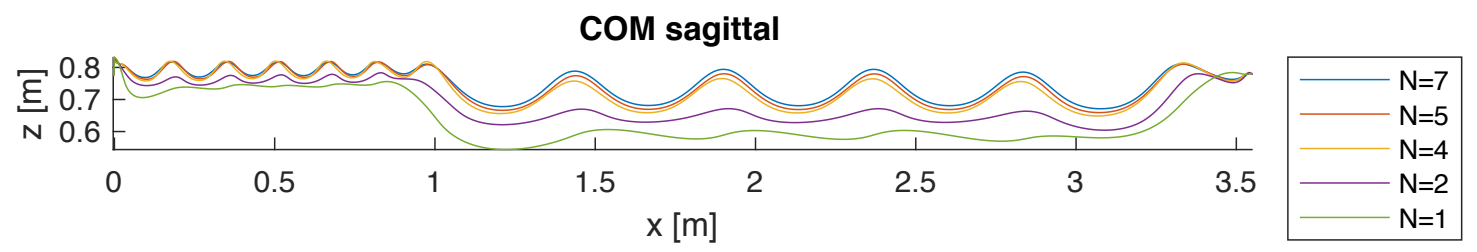

(a) Flat floor.

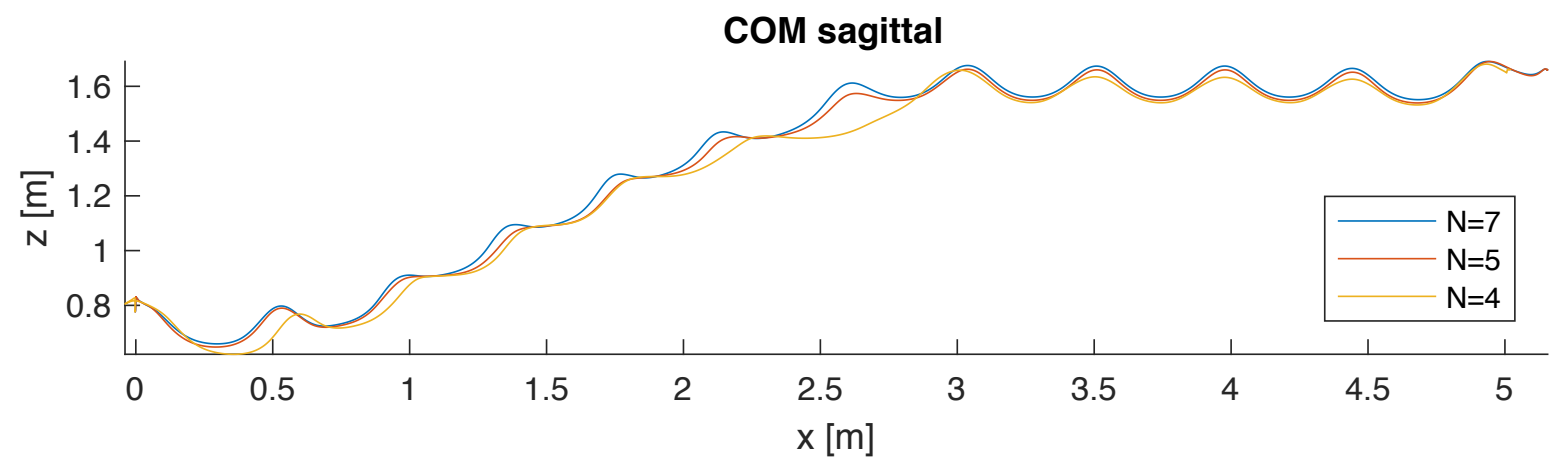

(b) Climbing stairs

Fig. 1: Sagittal view of CoM trajectories based on piecewise exponentials with different values of $N$.

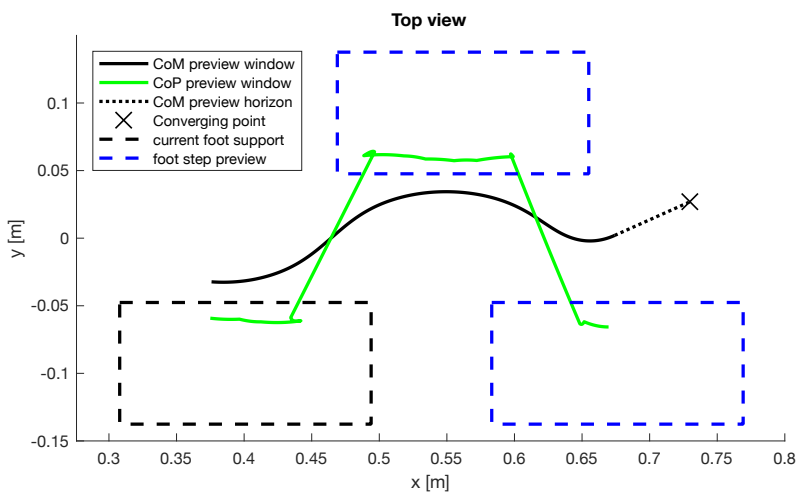

(a) Without 3D capturability.



(b) With 3D capturability.

Fig. 2: Snapshot of CoM and CoP trajectories over the whole prediction horizon at $\mathrm{t}=4.4 \mathrm{~s}$.

[9] J. Englsberger, C. Ott, and A. Albu-Schäffer, "Three-dimensional bipedal walking control using divergent component of motion," in Intelligent Robots and Systems (IROS), 2013 IEEE/RSJ International Conference on. IEEE, 2013, pp. 2600-2607.

[10] H. Dai and R. Tedrake, "Planning robust walking motion on uneven terrain via convex optimization," in Humanoid Robots (Humanoids), 2016 IEEE-RAS 16th International Conference on. IEEE, 2016, pp. 579-586.

[11] B. Ponton, A. Herzog, S. Schaal, and L. Righetti, "A convex model of humanoid momentum dynamics for multi-contact motion generation," in Proceedings of the IEEE-RAS International Conference on Humanoid Robots, 2016.

[12] C. Brasseur, A. Sherikov, C. Collette, D. Dimitrov, and P.-B. Wieber, "A robust linear mpc approach to online generation of $3 \mathrm{~d}$ biped walking motion," in Humanoid Robots (Humanoids), 2015 IEEE-RAS 15th International Conference on. IEEE, 2015, pp. 595-601.
[13] D. Serra, C. Brasseur, A. Sherikov, D. Dimitrov, and P.-B. Wieber, "A newton method with always feasible iterates for nonlinear model predictive control of walking in a multi-contact situation," in Humanoid Robots (Humanoids), 2016 IEEE-RAS 16th International Conference on. IEEE, 2016, pp. 932-937.

[14] R. J. Griffin and A. Leonessa, "Model predictive control for dynamic footstep adjustment using the divergent component of motion," in Robotics and Automation (ICRA), 2016 IEEE International Conference on. IEEE, 2016, pp. 1763-1768.

[15] S. Caron, A. Escande, L. Lanari, and B. Mallein, "Capturability-based pattern generation for walking with variable height," submitted, 2019.

[16] A. Del Prete, S. Tonneau, and N. Mansard, "Zero step capturability for legged robots in multicontact," IEEE Transactions on Robotics, no. 99 , pp. 1-14, 2018

[17] T. Koolen, M. Posa, and R. Tedrake, "Balance control using center of mass height variation: limitations imposed by unilateral contact," in 


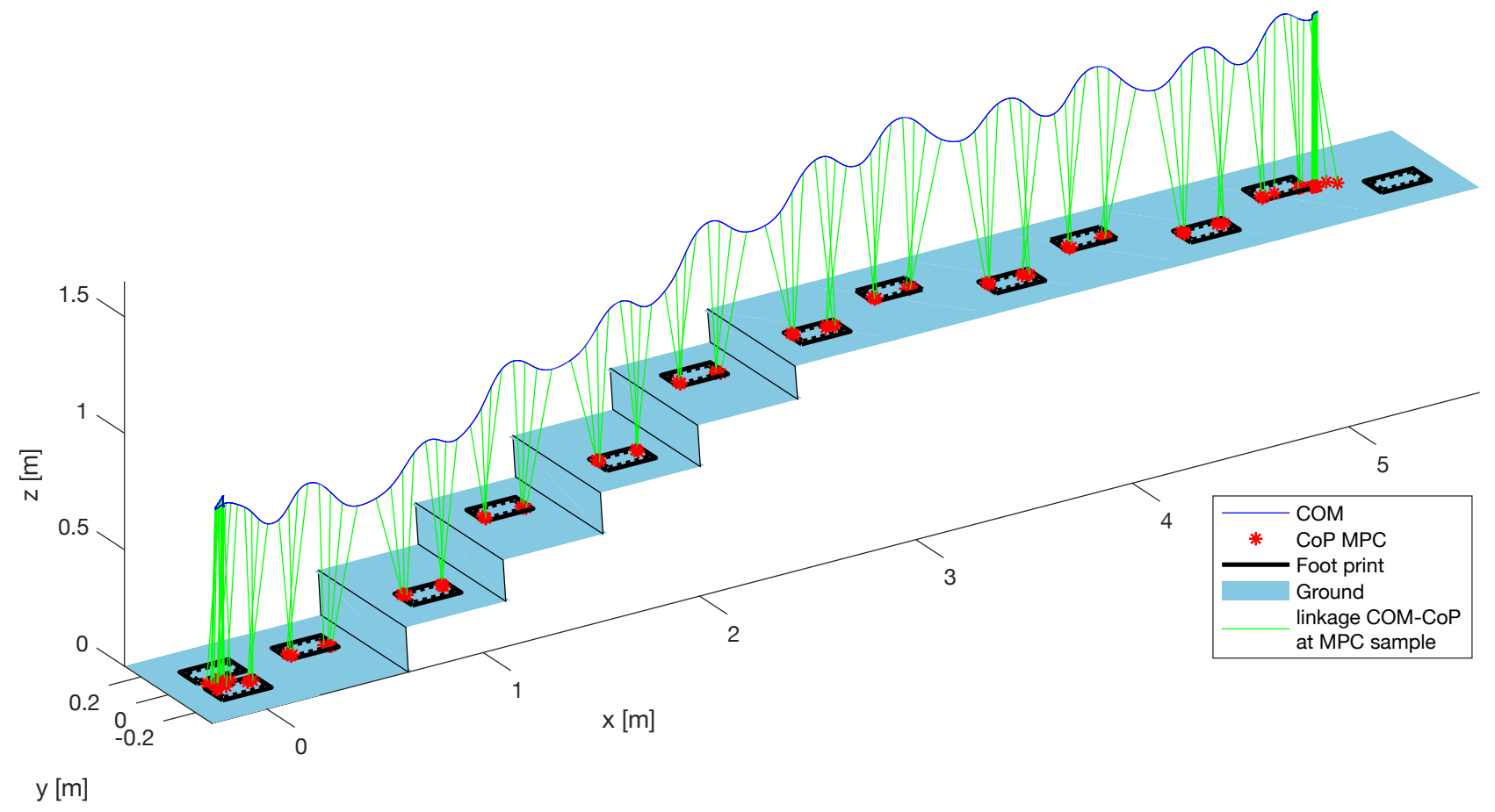

Fig. 3: 3D CoM trajectories when walking up stairs with the proposed linear MPC scheme.

International Conference on Humanoid Robots (Humanoids). IEEE, 2016, pp. 8-15.

[18] O. E. Ramos and K. Hauser, "Generalizations of the capture point to nonlinear center of mass paths and uneven terrain," in Humanoid Robots (Humanoids), 2015 IEEE-RAS 15th International Conference on. IEEE, 2015, pp. 851-858.

[19] P.-B. Wieber, S. Kuindersma, and R. Tedrake, "Modeling and control of legged robots," in Springer Handbook of Robotics, 2nd ed., B. Siciliano and O. Khatib, Eds. Springer Berlin Heidelberg, 2016, pp. 1203-1234.

[20] K. Kaneko, F. Kanehiro, M. Morisawa, K. Akachi, G. Miyamori, A. Hayashi, and N. Kanehira, "Humanoid robot HRP-4 - humanoid robotics platform with lightweight and slim body," in IEEE/RSJ International Conference on Intelligent Robots and Systems, 2011. 